\title{
Efficacy of an egress window to reduce the capture of undersize waved whelk (Buccinum undatum) in conical traps: laboratory experiments on the effect of vertical surge and trap angle during haul back.
}

\author{
Scott M. Grant \\ Centre for Sustainable Aquatic Resources \\ Marine Institute of Memorial University of Newfoundland \\ PO Box 4920, St. John's, Newfoundland, Canada A1C 5R3 \\ Email: scott.grant@mi.mun.ca
}

\begin{abstract}
Grant, S. M. 2013. Efficacy of an egress window to reduce the capture of undersize waved whelk (Buccinum undatum) in conical traps: laboratory experiments on the effect of vertical surge and trap angle during haul back. J. Northw. Atl. Fish. Sci., 45: 11-17. doi:10.2960/J.v45.m687
\end{abstract}

\begin{abstract}
The objective of this study was to develop a means to reduce the capture of undersize waved whelk (Buccinum undatum) in small mesh (51-64 mm) conical traps. To achieve this, egress windows were incorporated above the bottom ring of a trap to produce openings of sufficient width to allow undersize whelk to be released as a string of traps is being hauled to the surface. Analysis of the passage of undersize whelk through three egress window sizes revealed that a $30 \mathrm{~mm}$ wide window was most suitable. Simulations of two vertical surge distances and two trap angles were carried out in a controlled environment to provide a better understanding of trap performance. Experiments revealed that when a trap is oriented at an angle of $40-45^{\circ}$ a substantial percentage (29-36\%) of undersize whelk can be removed at vertical surge distances of $0.3-1 \mathrm{~m}$. Few (3-4\%) legal size whelk were emitted at a trap angle of $40-45^{\circ}$ and all whelk emitted from a trap were observed to pass through an egress window. Increasing the angle of the trap to $70-75^{\circ}$ resulted in loss of legal size whelk through the entrance located on top of the trap. The results of these simple experiments are promising but require verification through fishery trials before they can serve management decisions. Trap modifications and a trap line configuration that will maximize the egress of undersize whelk during fishing trials are discussed.
\end{abstract}

Keywords: Buccinum undatum; conical trap; egress window; undersize whelk

\section{Introduction}

The waved whelk (Buccinum undatum) is a small marine gastropod that occurs on both sides of the North Atlantic Ocean. Waved whelk represents a fairly large and recent fishery in the Newfoundland and Labrador region. In 2008, a competitive quota of $5000 \mathrm{t}$ was established in offshore waters of St. Pierre Bank (NAFO Subdivision 3Ps) and this fishery has accounted for approximately $90 \%$ of the landings within the region in recent years (DFO, 2011). Currently, annual landings of waved whelk remain high, but there are indicators that the resource is declining; average catch rates have decreased, fishermen are expanding the geographic range of effort to new grounds, and there is an increasingly large amount of small whelk being landed annually. Further, life-history characteristics of waved whelk make them particularly susceptible to over exploitation. Fecundity is low, eggs are laid on the ocean floor, and young hatch directly into a juvenile stage (Martel et al., 1986; Himmelman and Hamel, 1993). Direct development and a benthic habit leads to a much more limited dispersal than species with young that are released as pelagic free-floating larvae. Limited dispersal may not only lead to overfishing over small geographic scales, but also limit the renewal process as only whelk within the immediate area can replenish localized populations. This can be further exacerbated by unaccounted fishing mortality of large numbers of undersize juveniles.

The minimum legal size for waved whelk captured on the St. Pierre Bank is a shell length of $63 \mathrm{~mm}$ (DFO, 2011). The St. Pierre Bank trap line fishery uses conical traps with a bottom diameter of $95 \mathrm{~cm}$ and there are no regulations on minimum mesh size or the use of escape mechanisms. Traps are currently fitted with small mesh 
(51-64 mm) which facilitates the movement of whelk up the side and into the entrance located on top of the trap (Ram et al. 1993). This mesh size allows only the smallest of whelk ( $<35 \mathrm{~mm}$ shell length) to pass through the mesh opening during escapement or as a trap is being hauled back through the water column. Further, the conical frame construction results in a progressive reduction in the size of the opening of the diamond mesh from the lower to upper region of a trap. This limits the egress of even the smallest undersize whelk as a trap fills. Ultimately, the use of small mesh traps leads to the capture of substantial numbers of undersize whelk which has led to research into the development of an on board whelk grader that mechanically separates smaller whelk from the catch. Unfortunately, the use of mechanical devices can damage the shell and operculum and severe damage can lead to mortality from predation and disease (Mensink et al., 2000). Discarding of undersize individuals on unsuitable habitat may also negatively influence survival (Grati et al., 2010).

The movement of waved whelk towards a baited trap (Himmelman, 1988) and the effective area fished by a trap (McQuinn et al., 1988) have been investigated but selective properties of various mesh sizes or modifications to trap design to facilitate the egress of undersize waved whelk have not been studied. Lack of studies on the selective properties of waved whelk traps may in part be related to fishing practices in Atlantic Canada. Waved whelk traps are usually tended within 24-48 hours allowing little time for voluntary escapement of whelk. Further, in most instances there is still bait remaining in a trap when it is hauled, particularly where bait protection devices are used. Size selectivity experiments in gastropod trap fisheries with 24 hour soak times have demonstrated some success however it is not clear whether small individuals are able to escape voluntarily or if the main selection occurs during hauling procedures (Grati et al., 2010). Even size selectivity studies in a gastropod trap fishery with soak time of about one week found the best results were obtained from a trap that provided the added benefit of the egress of small whelk as they tumbled out of the trap during haul back (Park et al., 2007).

The current study used the same construction principal as the double hoop trap introduced by Park et al. (2007) to produce egress windows of uniform width around the bottom ring of a conical waved whelk trap. Experimental procedures combined studies to determine the egress window size and trap angle most suitable for the release of undersize whelk with minimal loss of legal size individuals. Experiments were carried-out in a controlled environment where the egress of whelk could be visually monitored during simulations of the vertical surge which occurs during haul back of a string of whelk traps.

\section{Materials and methods}

Fast frozen waved whelk captured on the St. Pierre Bank in autumn of 2009 were used in this study. The foot of frozen whelk was observed to be retracted in a manner commonly observed for live whelk when they are captured in trap fisheries. Once the foot is retracted shell-size dictates the optimal dimension of an egress window. Thus, egress experiments conducted with frozen whelk were considered to be a suitable proxy for live whelk. Shell length and shell width measurements $( \pm 1 \mathrm{~mm})$ were obtained by Vernier caliper for a total of 512 non-damaged waved whelk (Fig. 1). Least squares regression was used to examine the relationship between shell width and shell length.

Three egress window widths were studied based on regression analysis of the shell width $( \pm 5 \mathrm{~mm})$ at the maximum shell length of undersize waved whelk (i.e. $62 \mathrm{~mm}$ ). In an attempt to maximize the potential for release while still providing adequate area for whelk to crawl up the side of a trap a total of three egress windows of the same width were incorporated into a conical waved whelk trap by welding a steel bar between every second diagonal frame support (Fig. 2). The pots were fitted with $64 \mathrm{~mm}$ mesh netting which was extended around the bottom ring between each egress window to provide a means for whelk to crawl up on the trap (Fig. 2). Recapture
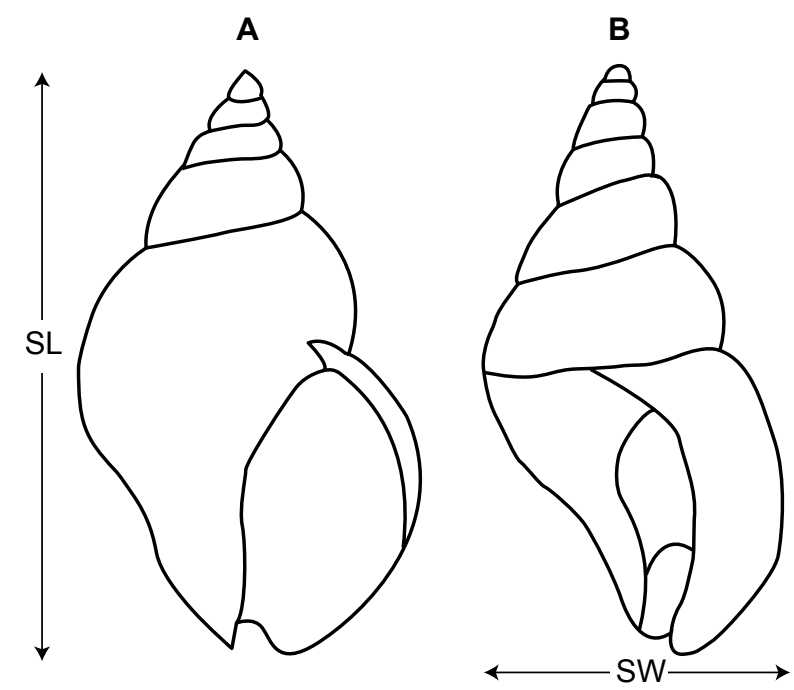

Fig. 1. Apertural view A) and lateral view B) of a waved whelk shell illustrating shell length (SL) and shell width (SW) measurements. 

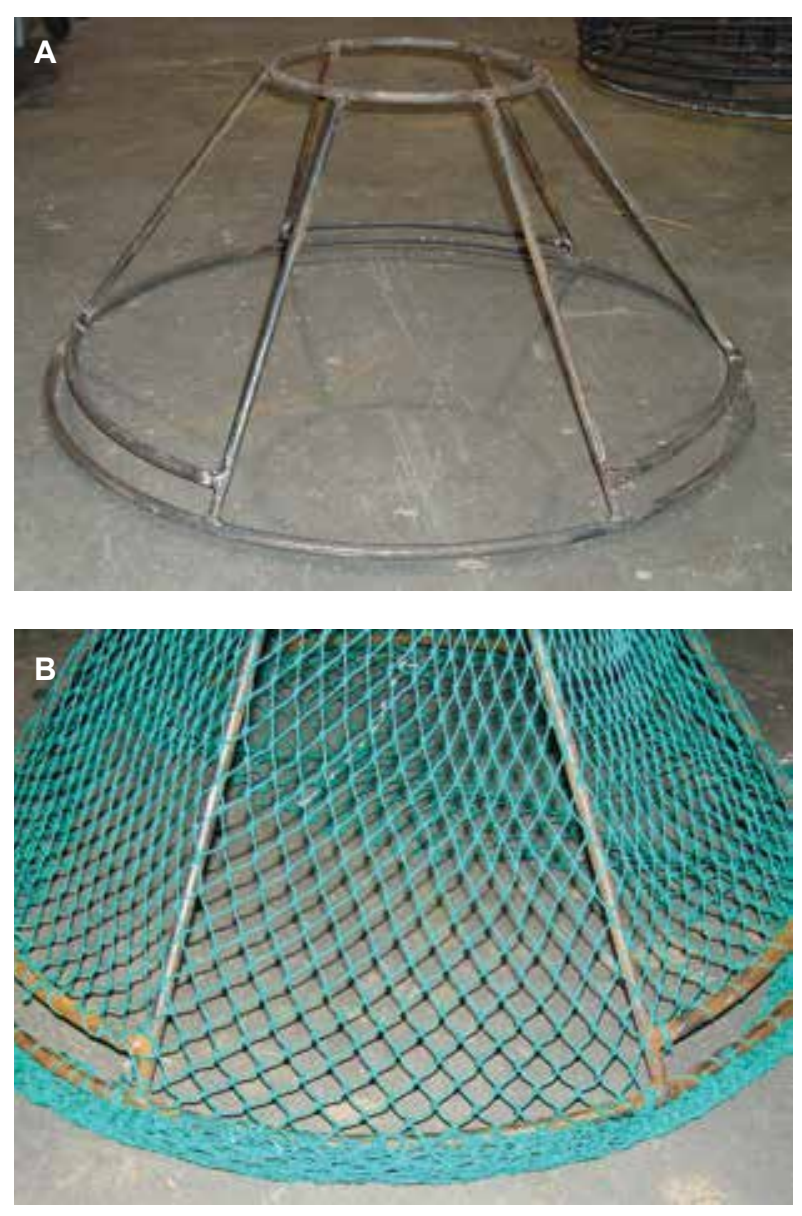

Fig. 2. Photographs of A) waved whelk trap frame showing egress windows at three locations above the bottom ring of a trap and $\mathbf{B}$ ) netting extending around the bottom ring of the trap between egress windows.

rate isopleths, obtained as a function of distance waved whelk traveled to a baited trap (Himmelman, 1988; McQuinn et al., 1988), demonstrate that a portion of the netting around the bottom ring of a modified trap will fall within the active space created by the bait plume.

Aerial dry-run tests of the performance of different size egress windows were conducted. This was accomplished by sorting frozen whelk into $10 \mathrm{~mm}$ shell length bins. The dry-run test procedure consisted of placing a sample of frozen whelk of known weight from each shell length bin into a modified trap. The trap was raised to a $70-75^{\circ}$ angle from horizontal and gently moved up and down until no whelk passed through an egress window. Whelk that passed through a window were weighed to obtain the percent removed. This procedure was repeated for each $10 \mathrm{~mm}$ shell length bin and egress window size.
Table 1. Contribution of each $10 \mathrm{~mm}$ shell length bin to the undersize waved whelk category.

\begin{tabular}{ccc}
\hline $\begin{array}{c}\text { Shell length bin } \\
(\mathrm{mm})\end{array}$ & $\begin{array}{c}\text { Weight } \\
(\mathrm{kg})\end{array}$ & $\begin{array}{c}\text { Percent of sub-legal } \\
\text { size category }\end{array}$ \\
\hline $31-40$ & 2.5 & 32.5 \\
$41-50$ & 2.3 & 29.9 \\
$51-60$ & 2.4 & 31.2 \\
$61-62$ & 0.5 & 6.5 \\
\hline Totals & 7.7 & \\
\hline
\end{tabular}

Vertical surge simulation experiments utilized a $23.7 \mathrm{~kg}$ sample of previously frozen whelk. The sample consisted of $7.7 \mathrm{~kg}$ of undersize $(<63 \mathrm{~mm})$ and $16 \mathrm{~kg}$ of legal size $(\geq 63 \mathrm{~mm}$ ) whelk. Thus, undersize whelk accounted for $32.5 \%$ of the sample. The contribution of each $10 \mathrm{~mm}$ length bin to the undersize category is summarized in Table 1 . The $23.7 \mathrm{~kg}$ sample was placed in a modified trap and lowered into a tank $(5.5 \mathrm{~m} \times 4.0 \mathrm{~m} \times 4.0 \mathrm{~m} ; \mathrm{L} \times$ $\mathrm{W} \times \mathrm{D}$ ) filled with freshwater. The tank possessed a large viewing window. A net bag was suspended under the trap to collect whelk that were emitted from the trap.

The vertical surge experiments tested the effect of the angle of the trap in the water column and the vertical distance a trap was raised and lowered in the water column (i.e., vertical surge distance). Two trap angle treatments (40-45 and $70-75^{\circ}$ from horizontal) and two vertical surge distance treatments $(0.3 \mathrm{~m}$ and $1 \mathrm{~m})$ were tested. During each experimental trial a trap was gently raised and lowered the vertical surge distance for two minutes with each surge cycle lasting approximately $8-10$ seconds. Once an experiment was complete the whelk in the net bag were retrieved and sorted into undersize and legal size categories and weighed. Three replicates were performed for each of the two trap angle and vertical surge distance treatments. Independent-samples $t$-tests were used to test for differences in the percentage of legal and undersize whelk removed from modified traps. An arcsine squareroot transformation was performed upon the data.

\section{Results}

Least squares regression analysis demonstrated a strong linear relationship between shell width and shell length of waved whelk (Fig. 3). The linear equation for the line of best fit (Fig. 3) indicates the maximum undersize shell length of $62 \mathrm{~mm}$ coincides with a shell width of $30.4 \mathrm{~mm}$. Therefore, the three egress window widths used in the dry-run tests were $25 \mathrm{~mm}, 30 \mathrm{~mm}$, and $35 \mathrm{~mm}$. 


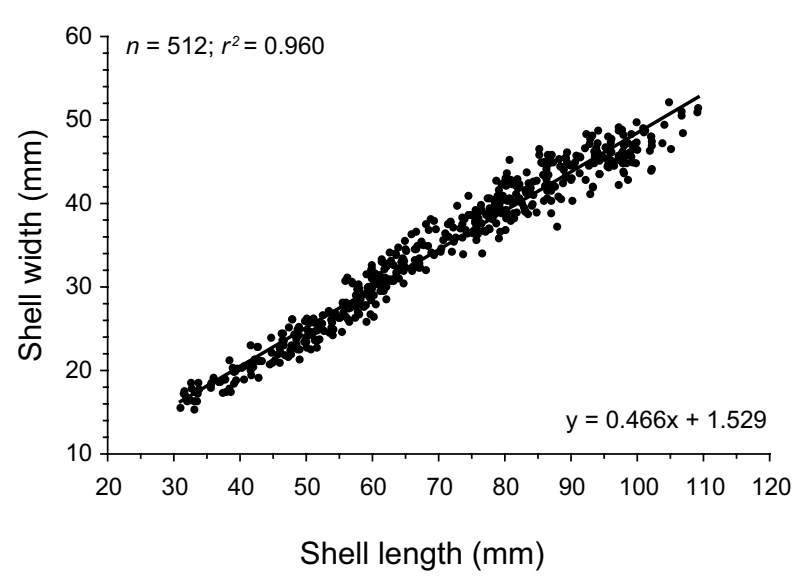

Fig. 3. Shell width to shell length relationship for St. Pierre Bank waved whelk. The total number of whelk examined $(n)$, correlation coefficeint $\left(r^{2}\right)$, and equation for the line of best fit is also shown.

During the dry-run tests it quickly became apparent that traps with egress window widths of $25 \mathrm{~mm}$ and $35 \mathrm{~mm}$ would not be suitable. The trap with a $25 \mathrm{~mm}$ egress window allowed individuals from only the smallest $10 \mathrm{~mm}$ shell length bins to be removed while 96-100\% of the whelk from the 61-70, 71-80, and 81-90 mm length bins were removed from the trap with the $35 \mathrm{~mm}$ egress window (Table 2). The $30 \mathrm{~mm}$ wide egress window exhibited the best performance, excluding all whelk from the $<60 \mathrm{~mm}$ bins, retained nearly $50 \%$ of the whelk from the $61-70 \mathrm{~mm}$ bin, and retained all whelk from the $>80 \mathrm{~mm}$ bins (Table 2). It was therefore decided that the vertical surge experiments would continue with the trap that possessed the $30 \mathrm{~mm}$ wide egress window.

The vertical surge experiments demonstrate that when as little as $32.5 \%$ of the catch is comprised of undersize whelk an average of $24.7-40.2 \%$ of these whelk can be removed (Table 3 ). Observations from the viewing window revealed that at a trap angle of $40-45^{\circ}$ whelk were emitted from the lower egress window only at both the $0.3 \mathrm{~m}$ or $1 \mathrm{~m}$ vertical surge distance. However, at a trap angle of $70-75^{\circ}$ whelk were emitted through the lower egress window and the entrance located on top of

Table 2. Summary of dry-run experiments to determine the most suitable egress window width to use in the vertical surge experiments.

\begin{tabular}{lrcrcr}
\hline $\begin{array}{c}\text { Width of egress } \\
\text { window }\end{array}$ & $\begin{array}{c}\text { Shell length bin } \\
(\mathrm{mm})\end{array}$ & $\begin{array}{c}\text { Weight retained } \\
(\mathrm{kg})\end{array}$ & $\begin{array}{c}\text { Weight retained } \\
(\mathrm{kg})\end{array}$ & $\begin{array}{c}\text { Weight retained } \\
(\mathrm{kg})\end{array}$ & $\begin{array}{c}\text { Percent } \\
\text { emitted }\end{array}$ \\
\hline $25 \mathrm{~mm}$ & $31-40$ & 2.51 & 1.87 & 0.64 & 25.5 \\
& $41-50$ & 2.62 & 2.33 & 0.29 & 11.1 \\
& $51-60$ & 5.72 & 5.44 & 0.28 & 4.9 \\
& $61-70$ & 2.49 & 2.49 & 0 & 0 \\
& $71-80$ & 2.99 & 2.99 & 0 & 0 \\
& $81-90$ & 3.46 & 3.46 & 0 & 0 \\
& $91-100$ & 3.84 & 3.84 & 0 & 0 \\
\hline $30 \mathrm{~mm}$ & $31-40$ & 2.51 & 0 & 2.51 & 100 \\
& $41-50$ & 2.62 & 0 & 2.62 & 100 \\
& $51-60$ & 5.72 & 0 & 5.72 & 46.6 \\
& $61-70$ & 2.49 & 1.33 & 1.16 & 12.0 \\
& $71-80$ & 2.99 & 2.63 & 0.36 & 0 \\
& $81-90$ & 3.46 & 3.46 & 0 & 0 \\
\hline $35 \mathrm{~mm}$ & $91-100$ & 3.84 & 3.84 & 0 & 100 \\
& $31-40$ & 2.51 & 0 & 2.51 & 100 \\
& $41-50$ & 2.62 & 0 & 2.62 & 100 \\
& $51-60$ & 5.72 & 0 & 5.72 & 100 \\
& $61-70$ & 2.49 & 0 & 2.49 & 95.7 \\
& $71-80$ & 2.99 & 0.13 & 2.86 & 96.2 \\
& $81-90$ & 3.46 & 0.13 & 3.33 & 36.7 \\
\hline $91-100$ & 3.84 & 2.43 & 1.41 & \\
\hline
\end{tabular}


GRANT: Efficacy of reducing the capture of undersize waved whelk (Buccinum undatum) in conical traps

Table 3. Summary of vertical surge experiments carried-out on a conical waved whelk trap with a $30 \mathrm{~mm}$ wide egress window. Percent emitted is specific to each whelk size category.

\begin{tabular}{|c|c|c|c|c|c|c|c|c|c|c|}
\hline \multirow[b]{2}{*}{$\begin{array}{c}\text { Trap } \\
\text { angle }\end{array}$} & \multirow[b]{2}{*}{$\begin{array}{l}\text { Vertical } \\
\text { surge } \\
\text { distance }\end{array}$} & \multirow[b]{2}{*}{$\begin{array}{c}\text { Whelk } \\
\text { size } \\
\text { category }\end{array}$} & \multicolumn{2}{|c|}{ Trial 1} & \multicolumn{2}{|c|}{ Trial 2} & \multicolumn{2}{|c|}{ Trial 3} & \multicolumn{2}{|c|}{ Mean } \\
\hline & & & $\begin{array}{c}\text { Weight } \\
\text { emitted } \\
(\mathrm{kg})\end{array}$ & $\begin{array}{l}\text { Percent } \\
\text { emitted }\end{array}$ & $\begin{array}{c}\text { Weight } \\
\text { emitted } \\
(\mathrm{kg})\end{array}$ & $\begin{array}{l}\text { Percent } \\
\text { emitted }\end{array}$ & $\begin{array}{c}\text { Weight } \\
\text { emitted } \\
(\mathrm{kg})\end{array}$ & $\begin{array}{l}\text { Percent } \\
\text { emitted }\end{array}$ & $\begin{array}{c}\text { Weight } \\
\text { emitted } \\
(\mathrm{kg})\end{array}$ & $\begin{array}{l}\text { Percent } \\
\text { emitted }\end{array}$ \\
\hline \multirow[t]{4}{*}{$40-45^{\circ}$} & $0.3 \mathrm{~m}$ & $<63 \mathrm{~mm}$ & 2.6 & 33.7 & 3.3 & 42.9 & 2.5 & 32.5 & 2.8 & 36.4 \\
\hline & & $\geq 63 \mathrm{~mm}$ & 0.5 & 3.1 & 0.4 & 2.5 & 0.5 & 3.1 & 0.5 & 3.1 \\
\hline & $1.0 \mathrm{~m}$ & $<63 \mathrm{~mm}$ & 2.0 & 26.0 & 2.4 & 31.2 & 2.2 & 28.6 & 2.2 & 28.6 \\
\hline & & $\geq 63 \mathrm{~mm}$ & 0.7 & 4.4 & 0.5 & 3.1 & 0.5 & 3.1 & 0.6 & 3.8 \\
\hline \multirow[t]{4}{*}{$70-75^{\circ}$} & $0.3 \mathrm{~m}$ & $<63 \mathrm{~mm}$ & 1.6 & 20.8 & 2.1 & 27.3 & 1.9 & 24.7 & 1.9 & 24.7 \\
\hline & & $\geq 63 \mathrm{~mm}$ & 0.8 & 5.0 & 1.1 & 6.9 & 0.7 & 4.4 & 0.9 & 5.6 \\
\hline & $1.0 \mathrm{~m}$ & $<63 \mathrm{~mm}$ & 2.9 & 37.7 & 3.1 & 40.3 & 3.2 & 41.6 & 3.1 & 40.2 \\
\hline & & $\geq 63 \mathrm{~mm}$ & 2.8 & 17.5 & 2.6 & 16.3 & 2.1 & 13.1 & 2.5 & 15.6 \\
\hline
\end{tabular}

the trap at both the $0.3 \mathrm{~m}$ and $1 \mathrm{~m}$ vertical surge distance. Observations at a trap angle of $40-45^{\circ}$ also revealed that most of the whelk were emitted from the lower egress window within the first minute of a trial.

At a trap angle of $40-45^{\circ}$ there was a low percentage $(3.1-3.8 \%)$ of legal size whelk emitted from the legal size component at both the $0.3 \mathrm{~m}$ and $1 \mathrm{~m}$ vertical surge distances (Table 3). Variability in shell width at length (Fig. 3) accounts for the passage of some of the smaller legal size whelk. There was also a low percentage (5.6\%) of legal size whelk emitted when the trap was oriented at an angle of $70-75^{\circ}$ and exposed to a vertical surge distance of $0.3 \mathrm{~m}$. However, the percentage of legal size whelk emitted at a trap angle of $70-75^{\circ}$ increased substantially to $15.6 \%$ when the vertical surge distance was increased to $1 \mathrm{~m}$. This increase is attributed to loss of whelk through the entrance of the trap.

Analysis of the effect of vertical surge distance at a trap angle of $40-45^{\circ}$ indicated there was no significant difference in the percentage of undersize $\left(t_{4}=2.189\right.$, $p=0.094)$ or legal size $\left(t_{4}=1.352, p=0.248\right)$ whelk emitted from a modified trap. Analysis of the effect of vertical surge distance at an angle of $70-75^{\circ}$ indicated a significantly higher percentage of both undersize $\left(t_{4}=6.729, p=0.003\right)$ and legal size $\left(t_{4}=6.992, p=0.002\right)$ whelk were emitted from the traps at the higher vertical surge distance of $1 \mathrm{~m}$. Overall, these results indicate that increased vertical surge distances associated with inclement weather conditions will have a significant effect on the removal of both undersize and legal size whelk when a modified trap is oriented at an angle of $70-75^{\circ}$.

\section{Discussion}

Undersized waved whelk are highly susceptible to capture in baited traps with reports of undersize individuals accounting for as much as $80 \%$ of the catch in conical traps. Onboard sorting of large quantities of undersize whelk is a challenge and can inadvertently result in variable quantities of undersize individuals being landed. The mesh size of conical traps used in the St. Pierre Bank fishery is not regulated and studies on mesh size selectivity have not been undertaken to reduce the retention of undersize individuals. In the current study, the egress windows incorporated into a conical waved whelk trap were successful at removing a substantial percentage of undersize whelk under controlled conditions where the mechanism by which the whelk were emitted could be visually observed. Observations under controlled conditions not only lower the cost of evaluating new gear designs at sea, but also provide the opportunity to investigate specific features and performance of modified gears (Winger et al., 2006). The ability to make direct observations of the egress of whelk from a trap was important as modified traps will need to be affixed to the mainline so that a trap is oriented at an angle during haul back in order to expose whelk to the egress window. In the absence of direct observations, the loss of legal size whelk through the entrance would have gone undetected at a trap angle of $70-75^{\circ}$ and there would have been uncertainty with regard to the loss of whelk at a trap angle of $40-45^{\circ}$. These findings indicate that while a substantial loss of legal size whelk can occur through the entrance at a trap angle of $70-75^{\circ}$, there was no loss of whelk through the entrance at a trap angle of $40-45^{\circ}$. 
In the current study, a conical whelk trap was raised and lowered in the water column to simulate a vertical surge during haul back. Waved whelk fishermen indicate vertical surge distances of $0.3 \mathrm{~m}$ are common during haul back and vertical surges intermittently expose new whelk to the egress window increasing the probability of egress of undersize individuals. The experiments also simulated a vertical surge duration of two minutes. The period of time a trap will be exposed to vertical surges during haul back in a bottom trap line fishery will primarily depend upon the number of traps in the water column which is a function of bottom depth and distance between traps. However, bottom trap lines generally do not hang vertically during haul back so the number of traps in the water column tends to exceed the bottom depth to distance between traps ratio. Bottom depth ranges from about $60-80 \mathrm{~m}$ for most of the St. Pierre Bank (Dalrymple et al., 1992) and whelk are captured to depths of up to $180 \mathrm{~m}$ in Atlantic Canada (Kenchington and Glass, 1998). A vertical surge in a trap line will occur as the pitch and roll of the vessel is transferred to the trap line when the power to the hauler is disengaged to allow the contents of a trap to be dumped and sorted. The greater the number of traps in the water column the greater will be the effect of vertical surges at exposing undersize whelk to an egress window.

Distance between whelk traps in the St. Pierre Bank trap line fishery is variable with industry typically using the same trap line attachment configuration utilized in the snow crab fishery where the attractive distance to a baited trap has been estimated at about $37 \mathrm{~m}$ (E. Dawe, pers. comm., Fisheries and Oceans Canada). Studies of the movements of waved whelk toward a baited trap indicate shorter attractive distances of 10-18 m over a 24-48 hour period, respectively (Himmelman, 1988). Effective area fished by a baited waved whelk trap over a 24 hour period was highly variable with estimates ranging between $18-278 \mathrm{~m}^{2}$ and was found to depend on current speed and direction, depth, and seasonal variations in feeding rates and reproductive activity (Himmelman, 1988; McQuinn et al., 1988). Overall, studies of the movements of waved whelk toward a baited trap suggest the distance between traps in the St. Pierre Bank trap line fishery could be reduced to $18 \mathrm{~m}$ without negatively influencing catch rates. This reduction would expose at least three to four traps to a vertical surge over most of the St. Pierre Bank waved whelk fishing grounds during haul back of a trap line.

Susceptibility of undersize gastropods to capture in small mesh baited traps (Ram et al. 1993; Park et al. 2007; Grati et al. 2010) makes them highly vulnerable to overfishing and the percent contribution of undersize individuals will increase as the biomass of legal size individuals decreases.
Results presented in the current study show that egress windows are unlikely to remove all undersize whelk from a conical trap. The proportion of undersize whelk emitted from an egress window will not only depend on total catch weight but also the contribution and size distribution of undersize whelk to the catch. In the current study, observations of the passage of waved whelk through the egress window showed that the effect of vertical surge duration was largely limited to the first minute which is also likely to be influenced by catch characteristics. Catch rates of 9-16 kg of legal size whelk per trap are considered to be satisfactory in the St. Pierre Bank fishery. The current study simulated the upper range of catch rates for legal size whelk however, the contribution of undersize whelk is considered to be at about the midpoint between the lower and upper range commonly reported in commercial catches.

This study has demonstrated that at sea experiments on the efficacy of egress windows to reduce the catch of undersize individuals are warranted. Indeed, the results require verification through fishery trials before they can serve management decisions. Although three egress windows were used in the current study whelk were only emitted from the lower egress window when a trap was oriented at an angle of $40-45^{\circ}$. Therefore it is advised that a single egress window be used in future studies. Use of a single window would also likely limit the entry of undersize whelk through the egress window. During fishery trials it is advisable to attach modified traps to the mainline at $18 \mathrm{~m}$ intervals and verify the method of attachment orients the traps at an angle of $40-45^{\circ}$ during haul back. To achieve a $40-45^{\circ}$ angle, traps will require a simple modification of the bridle attachment from the mainline. Use of three points of attachment from the bridle to the top of the trap and reducing the length of the attachment that is centered over the egress window will achieve the desired trap angle and expose whelk to the egress window during haul back. Members of the fishing industry should be an integral component of future fishery trials as they will be the best judge of the efficacy of the egress window and operational limitations of modifications to the trap line configuration.

Conical traps are ideal for commercial applications because they take up less space than square or rectangular traps when they are stacked allowing several traps to be carried during a fishing trip. This is an important consideration in extended trips associated with offshore fisheries. Construction of a conical trap with an egress window requires only a minor modification over the current trap design and addition of an egress window does not influence storage capacity. 
Damage to the shell and operculum of whelk could occur during the capture and sorting process and the effects of coming in contact with and passing through an egress window are unknown. Anti-chaffing rope is used around the bottom ring of commercial traps to protect the netting. Extending the use of anti-chaffing rope around the egress window frame will reduce the potential for shell and operculum damage during a vertical surge. Nevertheless, studies of the unaccounted fishing mortality are recommended and should be comprehensive, identifying haul back and onboard sorting, grading, and discard processes that are most likely to result in shell and operculum damage that leads to mortality.

\section{Acknowledgements}

This study was funded by the Canadian Centre for Fisheries Innovation and Newfoundland and Labrador Department of Fisheries and Aquaculture. HSF Ocean Products Ltd. provided the frozen whelk and North Atlantic Marine Supplies and Services Inc. provided the trap frames and netting. Technical support was provided by Rennie Sullivan, Rebecca Sheppard, Matthew Sweeney, Andrew Thorne and Denise Wilcott. Wade Hiscock and an anonymous reviewer provided valuable comments on an earlier version of this manuscript.

\section{References}

DALRYMPLE, R. W., E. M. LCGRESLEY, G. B. J. DALRYMPLE, R. W., E. M. LCGRESLEY, G. B. J. FADER and B. D. PETRIE. 1992. The western Grand Banks of Newfoundland: Transgressive Holocene sedimentation under the combined influence of waves and currents. Mar. Geol., 105: 95-118. http://dx.doi.org/10.1016/0025$\underline{3227(92) 90184-\mathrm{I}}$

DFO 2011. Subdivision 3Ps offshore whelk: a preliminary assessment of male size at maturity. DFO Can, Sci. Advis. Sec. Sci. Advis. Rep., 2011/023, 8 p.
GRATI, F., P. POLIDORI, G. SCARELLA and G. FABI. 2010. Estimation of basket trap selectivity for changeable nassa (Nassarius mutabilis) in the Adriatic Sea. Fish. Res., 101: 100-107. http://dx.doi.org/10.1016/j.fishres.2009.09.012

HIMMELMAN, J. H. 1988. Movements of whelks (Buccinum undatum) towards a baited trap. Mar. Biol., 97: 521-531. http://dx.doi.org/10.1007/BF00391048

HIMMELMAN, J. H. and J. R. HAMEL. 1993. Diet, behaviour and reproduction of the whelk Buccinum undatum in the northern Gulf of St. Lawrence, eastern Canada. Mar. Biol., 116: 423-430. http://dx.doi.org/10.1007/BF00350059

KENCHINGTON, E. and A. GLASS. 1998. Local adaptation and sexual dimorphism in the waved whelk (Buccinum undatum) in Atlantic Nova Scotia with applications to fisheries management. Can. Tech. Rep. Fish. Aquat. Sci., No. 2237. 43 p.

MARTEL, A., D. H. LARRIVÉE and J. H. HIMMELMAN. 1986. Behaviour and timing of copulation and egg -laying in the neogastropod Buccinum undatum L. J. Exp. Mar. Biol. Ecol., 96: 27 -42.

$M^{\mathrm{C}}$ QUINN, I. H., L. GENDRON and J. H. HIMMELMAN. 1988. Area of attraction and effective area fished by a whelk (Buccinum undatum) trap under variable conditions. Can. J. Fish. Aquat. Sci., 45: 2054-2060. http://dx.doi.org/10.1139/ f88-239

MENSINK, B. P., C. V. FISHER, G. C. CADÉE, M. FONDS, C. C. TEN HALLER-TJABBES and J. P. BOON. 2000. Shell damage and mortality in the common whelk (Buccinum undatum) caused by beam trawl fishing. Jour. of Sea Res., 43: 53-64. http://dx.doi.org/10.1016/S1385-1101(00)00003-4

PARK, H., R. MILLAR, H. AN and H. KIM. 2007. Size selectivity of drum-net traps for whelk in the Korean coastal waters of the east sea. Fish. Res., 86: 113-119. http://dx.doi. org/10.1016/j.fishres.2007.05.008

RAM, N., K. NASHIMOTO, K. YAMAMOTO, and T. HIRAISHI. 1993. Size selectivity and catching efficiency of three different traps for whelks Neptunea arthritica in Shiriuchi, Hokkaido. Nippon Suisan Gakk., 59: 1313-1318. http:// dx.doi.org/10.2331/suisan.59.1313

WINGER, P. D., H. DELOUCHE, and G. LEGGE. 2006. Designing and testing new fishing gears: the value of a flume tank. Mar. Tech. Soc. Jour., 40: 44-49. http://dx.doi. org/10.4031/002533206787353240 
